\title{
Enhanced autophagy promotes the clearance of Pseudomonas aeruginosa in diabetic rats with wounds
}

\author{
Jun $\mathrm{Xu}^{1 \#}$, Yanan $\mathrm{Ma}^{2 \#}$, Xiaoqing Zhu ${ }^{3}$, Jinghang Zhang ${ }^{1}$, Zhihui Cheng ${ }^{4}$, Weihui Wu ${ }^{5}$, Penghua Wang ${ }^{1}$ \\ ${ }^{1}$ NHC Key Laboratory of Hormones and Development (Tianjin Medical University), Tianjin Key Laboratory of Metabolic Diseases, Department of \\ Diabetic foot, Tianjin Medical University Chu Hsien-I Memorial Hospital \& Tianjin Institute of Endocrinology, Tianjin, China; ${ }^{2}$ Fengyang College, \\ Shanxi Medical University, Fenyang, China; ${ }^{3}$ Department of Endocrinology, Qingdao Eighth People’s Hospital, Qingdao, China; ${ }^{4}$ Key Laboratory \\ of Molecular Microbiology and Technology of the Ministry of Education, Department of Microbiology, College of Life Sciences, Nankai University, \\ Tianjin, China; ${ }^{5}$ State Key Laboratory of Medicinal Chemical Biology, Key Laboratory of Molecular Microbiology and Technology of the Ministry \\ of Education, Department of Microbiology, College of Life Sciences, Nankai University, Tianjin, China \\ Contributions: (I) Conception and design: J Xu, Y Ma, Z Cheng, W Wu, P Wang; (II) Administrative support: Z Cheng, W Wu, P Wang; (III) \\ Provision of study materials or patients: Z Cheng, W Wu; (IV) Collection and assembly of data: J Xu, Y Ma, X Zhu, J Zhang; (V) Data analysis and \\ interpretation: J Xu, Y Ma, P Wang; (VI) Manuscript writing: All authors; (VII) Final approval of manuscript: All authors. \\ \#These authors contributed equally to this work. \\ Correspondence to: Panghua Wang. NHC Key Laboratory of Hormones and Development (Tianjin Medical University), Tianjin Key Laboratory of \\ Metabolic Diseases, Department of Diabetic foot, Tianjin Medical University Chu Hsien-I Memorial Hospital \& Tianjin Institute of Endocrinology, \\ Tianjin, China. Email: wph200000@163.com.
}

Background: To investigate the effects of the Pseudomonas aeruginosa (P. aeruginosa) type 3 secretion system (T3SS) on diabetic wound healing and autophagy-associated proteins.

Methods: P. aeruginosa and P. aeruginosa without T3SS were used to infect back wounds in 36 rats (18 normal and 18 diabetic). Followed infection with $P$. aeruginosa, another 36 rats (18 normal and 18 diabetic) with back wounds were treated with autophagy inducer rapamycin or gentamicin (positive control). Wound healing, colony count, $\mathrm{HE}$ and Masson staining were recorded. Western blot and immunofluorescent were used to determine the expression of the autophagy markers (LC3, beclin-1 and p62) in wound tissues.

Results: The number of $P$. aeruginosa colonies in infected wounds began to decrease on day 3 in normal rats and on day 7 in diabetic rats. The decrease was more apparent in P. aeruginosa without T3SS. The expression of LC3-II/LC3-I and beclin-1 gradually increased, and p62 gradually decreased in the wounds in of all groups; however, the changes were more dramatic in normal rats compared with diabetic rats on day 14. Rapamycin increased LC3-II/LC3-I and beclin-1 expression, and decreased p62 expression, gentamicin had no effect on their expression of autophagy markers.

Conclusions: T3SS of $P$. aeruginosa inhibited the entire autophagy process in wounds. Thus inducing autophagy could enhance the clearance of P. aeruginosa in diabetic wounds and is expected to become a new method of anti-P. aeruginosa infection.

Keywords: Autophagy; diabetic wound; Pseudomonas aeruginosa; rapamycin; type 3 secretion system (T3SS)

Submitted Jan 24, 2020. Accepted for publication Sep 06, 2020.

doi: $10.21037 /$ atm-20-1019

View this article at: http://dx.doi.org/10.21037/atm-20-1019

\section{Introduction}

It is estimated that by 2035 , the worldwide diabetes population will reach 600 million with approximate $80 \%$ of the patients residing in developing countries (1). Diabetic foot infection (DFI) is one of the most important causes of deterioration, amputation and death, as well as a common cause of high medical expenses for diabetic patients (2). Therefore, it is significant that we develop therapeutic 
strategies for patients with DFI.

Many recent studies have shown that Pseudomonas aeruginosa ( $P$. aeruginosa) is a common Gram-negative bacterium involved in DFI, especially in warm climates in Asia and Africa (3-5). In China, a recent study showed that the main pathogens of DFI were Gram-negative bacteria, including $P$. aeruginosa and were associated with long-term hospitalization and antibiotic use of diabetic patients. $P$. aeruginosa has been reported more and more to develop multidrug resistance Pseudomonas aeruginosa (MDRPA) (6). The type 3 secretion system (T3SS) is a significant pathogenic factor of $P$. aeruginosa. A major pathogenic mechanism is that $P$. aeruginosa directly injects exotoxins into host cells through the T3SS membrane channel, which causes cell necrosis and tissue destruction, facilitates bacterial invasion and diffusion, and escaping from phagocytosis $(7,8)$.

Recent studies have reported that autophagy plays a principal role in the defense systems of infectious pathogens. Pathogens can produce multiple virulence factors and toxins that interfere with autophagy, in which T3SS plays an outstanding role (9).

Our previous study showed that the expression of the autophagy markers [microtubule-associated protein light chain 3 (LC3) and beclin-1] was decreased in the granulation tissues of diabetic foot wounds infected with $P$. aeruginosa, and was even lower in infections with MDRPA (10). The objective of our study was is to determine whether the T3SS of $P$. aeruginosa affected the autophagy of cells in wound and whether $P$. aeruginosa could be eliminated by changing the host autophagy level of host. The results would offer a new possibility for the clinical treatment of DFI.

We present the following article in accordance with the ARRIVE reporting checklist (available at http://dx.doi. org/10.21037/atm-20-1019).

\section{Methods}

\section{Subjects}

\section{Animals and bacterial strains}

Seventy-two clean-grade, 7-week-old male Sprague-Dawley (SD) rats (190-210 g) were purchased from Beijing Hua Fukang Biotechnology Co., Ltd. (Beijing, China). The rats were housed in a specific-pathogen-free grade animal room at Tianjin Medical University, and all experiments were conducted in the microbiology laboratories at Tianjin Medical University General Hospital or Tianjin Medical
University Chu Hsien-I Memorial Hospital (Metabolic Disease Hospital). Experiments were performed under a project license (No.: SCXK2019-0008) granted by the Ethics Committee of Tianjin Medical University, in compliance with the guidelines of Ethics Committee of Tianjin Medical University for the care and use of animals.

The $P$. aeruginosa and $P$. aeruginosa without T3SS $(P$. aeruginosa $\triangle$ pcrV: mutant excluding the T3SS structure) were obtained from Associate Professor Zhihui Cheng (Department of Microbiology, Nankai University).

\section{Experimental methods}

\section{Isolation and culture of bacteria}

$P$. aeruginosa and $P$. aeruginosa without T3SS were inoculated onto LB agar plates and incubated at $37{ }^{\circ} \mathrm{C}$. After 24 h, single colony was cultured in LB liquid medium overnight with shaking. Liquid cultures were centrifugated at 3,000 rpm for $2 \mathrm{~min}$, and the supernatant was removed. The pellets were washed twice with normal saline and spun twice-then the supernatant was removed. The bacteria were resuspended in normal saline, mixed well by pipetting-and the bacterial concentrations were adjusted to $1 \times 10^{8}$ cells $/ \mathrm{mL}$ using a spectrophotometer. Approximate $10^{7}$ bacteria (100 $\mu \mathrm{L}$ suspension) were inoculated onto each wound.

\section{Experimental animal grouping}

The thirty-six 7-week-old SD rats were randomly divided into six groups using a random number table: diabetes mellitus uninfected group (DM + uninfected), diabetes mellitus $P$. aeruginosa-infected group (DM $+P$. aeruginosa), diabetes mellitus $P$. aeruginosa without T3SS infected group $(\mathrm{DM}+P$. aeruginosa $\triangle \mathrm{pcrV})$, normal uninfected group $(\mathrm{N}$ + uninfected), normal $P$. aeruginosa infected group $(\mathrm{N}+P$. aeruginosa), and normal $P$. aeruginosa without T3SS infected group $(\mathrm{N}+P$. aeruginosa $\triangle \mathrm{pcrV})$.

The other thirty-six 7-week-old SD rats were randomly divided into six groups using a random number table: diabetes mellitus $P$. aeruginosa infected group (DM $+P$. aeruginosa), diabetes mellitus $P$. aeruginosa infected and rapamycin intervention group $(\mathrm{D} M+P$. aeruginosa + rapamycin), normal $P$. aeruginosa-infected group $(\mathrm{N}+P$. aeruginosa), normal $P$. aeruginosa infected and rapamycin intervention group $(\mathrm{N}+P$. aeruginosa + rapamycin $)$, diabetes mellitus $P$. aeruginosa infected and gentamicin intervention group $(\mathrm{DM}+P$. aeruginosa + gentamicin $)$, and normal $P$. aeruginosa-infected and gentamicin intervention group $(\mathrm{N}+$ $P$. aeruginosa + gentamicin). 
Establishment of a type $\mathbf{2}$ diabetes rat model

The rats designated for experimental diabetes induction were fed a high-fat and high-sugar diet, while rats designated as the normal groups were fed a standard diet. The rats were given 12 hours lights/12 hours dark cycle. The room temperature was controlled between $20-25^{\circ} \mathrm{C}$. After 8 weeks, all rats were given fasting with water ad libitum for 12 hours before modeling. Rats in the diabetes mellitus groups were intraperitoneally injected with $1 \%$ streptozotocin (Sigma, St. Louis, MO, USA) at a dose of $35 \mathrm{mg} / \mathrm{kg}$, and rats in the normal groups were intraperitoneally injected with the same volumes of citric acid buffer. After 7 days, blood samples were obtained from the tail vein to measure blood glucose level. A random blood glucose $>16.7 \mathrm{mmol} / \mathrm{L}$ was confirmed a diabetes model had been established.

\section{Establishment of an infected wound model in diabetic rats}

Rats were anesthetized by intraperitoneal injection of $10 \%$ chloral hydrate and full thickness ulcers of $1 \mathrm{~cm}$ in diameter were made on both the left and right sides of the back using a perforator. Rats were infected with $P$. aeruginosa or $P$. aeruginosa $\triangle \mathrm{pcrV}$ and subsequently fed in individual cages. Wound healing was observed and colony counting was performed on days $0,3,7,14$ and 21 . Wound tissues were obtained on days 0,7 and 14 .

\section{Rapamycin and gentamicin intervention}

Rapamycin (MedChemExpress, Monmouth Junction, NJ, USA) was diluted to $1 \mathrm{mg} / \mathrm{mL}$ with sterilized water containing $5.2 \%$ Tween 80 and $5.2 \%$ polyethylene glycol 400. After the infection model was established, $2.5 \mathrm{mg} / \mathrm{kg}$ of rapamycin was injected intraperitoneally once daily on days $1-5$.

After the infection model was established, $100 \mathrm{mg} / \mathrm{kg}$ of gentamicin (Sinopharm Chemical Reagent Co., Ltd, Shanghai, China) was injected intramuscularly once daily on days 1-3.

\section{HE staining of wound tissue}

The paraffin section was baked in an oven at $60^{\circ} \mathrm{C}$ for $1 \mathrm{~h}$. Dewaxing in xylene and alcohol. Hematoxylin stained for $1 \mathrm{~min}$, color separated in 1\% hydrochloric acid alcohol for $3 \mathrm{~s}$, PBS washed then warm water washed to make the nucleus return to blue. Eosin stained for 2 min-then PBS washed. After alcohol dehydration the section was put into xylene-and finally the neutral gum was dropped into the tissue to seal the section. Observed the wound tissues under optical microscope.

\section{Masson staining of wound tissue}

The paraffin section was dewaxed to water, stained with hematoxylin for $5 \mathrm{~min}$. Color separated in $1 \%$ hydraulic acid alcohol for $3 \mathrm{~s}$ and washed with distilled water. It was dyed with Masson Fuchsin acid solution for $8 \mathrm{~min}$. Then the section was washed with $2 \%$ glacial acetic acid for $5 \mathrm{~s}$, differentiated for 3 min with $1 \%$ phosphomolybdic acid solution. Next it was stained with aniline blue for $5 \mathrm{~min}$ then was washed with $0.2 \%$ glacial acetic acid solution for 5 s. Finally, it was washed with $95 \%$ alcohol, anhydrous alcohol, transparented with xylene-and sealed with neutral gum. Observed the fiber tissues under optical microscope.

\section{Immunohistochemical detection of the expression of LC3, beclin-1, and p62 in wound tissue}

Under the light microscope, there were brown granules in the cytoplasm, which represented the staining sites of LC3 or beclin-1 or p62. The mean optical density (MOD) of LC3 or beclin-1 or p62 was analyzed by Image. ProPlus 6.0. Five high power fields (400x) were randomly selected from each section, and the mean value of the five fields was taken as the final MOD value of each section.

\section{Western blot analysis of LC3-II/LC3-I, beclin-1 and p62 expression}

Wound tissue was ground with radioimmunoprecipitation assay buffer then centrifugated at $4{ }^{\circ} \mathrm{C}$ at $12,000 \mathrm{rpm}$ for $10 \mathrm{~min}$. After denaturation of the proteins, electrophoresis was carried out at $60 \mathrm{~V}$ for approximately $30 \mathrm{~min}$, followed by $120 \mathrm{~V}$ for approximately $1 \mathrm{~h}$ after the lowest marker entered the separation gel. Protein bands were blotted onto a polyvinylidene difluoride membrane at a constant current of $250 \mathrm{~mA}$. After blotting, the membrane was incubated with primary antibodies against $\beta$-actin, LC3, beclin-1 and p62 ( $\beta$-actin: Beijing Solarbio Science \& Technology Co., Ltd., Beijing, China; LC3: Abcam, Cambridge, UK; beclin-1: Abgent, San Diego, CA, USA; and p62: Cell Signaling Technology, Danvers, MA, USA). All antibodies were diluted at 1:1000 and incubated overnight at $4{ }^{\circ} \mathrm{C}$ under gentle shaking. Membranes were incubated with secondary antibodies $(1: 1,000$ to $1: 2,000)$ at room temperature for $1 \mathrm{~h}$. Protein bands were visualized using enhanced chemiluminescence and the protein levels were quantified using the gray value ratio of the target protein to $\beta$-actin. 
Double immunofluorescent staining of LC3 expression in macrophages of wound tissue

Paraffin-embedded slices of wound tissue were incubated at $60{ }^{\circ} \mathrm{C}$ for $1 \mathrm{~h}$, then cooled at room temperature for $30 \mathrm{~min}$. The slices were de-waxed to transparency with xylene, soaked in an ethanol gradient $(100 \%, 95 \%, 90 \%, 80 \%$ and $70 \%)$ for 5 min each then trice-washed with phosphate-buffered saline. Slices were then placed in citric acid buffer $(\mathrm{pH}$ 6.0) and incubated for $5 \mathrm{~min}$ at medium power in a microwave oven for antigen retrieval. Slices were cooled at room temperature for $10 \mathrm{~min}$, then $50 \mu \mathrm{L}$ of $0.5 \%$ Triton $\mathrm{X}-100$ was added to each slice and incubated for $5 \mathrm{~min}$. For blocking, $50 \mu \mathrm{L}$ goat serum was added to each slice and incubated at room temperature for $1 \mathrm{~h}$, then $50 \mu \mathrm{L}$ of LC3 (diluted 1:100) and $50 \mu \mathrm{L}$ of CD14 (1:100; Abgent) were added onto each slice for incubation in a light-shielded wet box for $12 \mathrm{~h}$ at $4^{\circ} \mathrm{C}$ and reheated at $37^{\circ} \mathrm{C}$ for $45 \mathrm{~min}$. Rabbit IgG antibody labeled with fluorescein isothiocyanate and anti-mouse IgG antibody labeled with Alexa Fluor 594 (both from Cell Signaling Technology) were diluted 1:500 and added to each slice, and incubated at room temperature for $1 \mathrm{~h}$ under darkness, followed by adding $50 \mu \mathrm{L}$ of nuclear staining agent DAPI (4'6-diamidino-2-phenylindole; Cell Signaling Technology) for a further 2 min incubation under darkness. An adequate amount of anti-fluorescence quenching reagent was added to each slice on a glass slide and covered. The results of immunofluorescence staining were observed under confocal microscope as follows: LC3, bright green; macrophage marker CD14, bright red; co-localized LC3 and CD14, yellow; and DAPI stained nuclei, blue.

\section{Statistical analysis}

Quantitative data are expressed as the mean \pm SD. Qualitative data are expressed as a number (percent). Multivariate analysis of variance was used to compare the repeated quantitative data. The least significant difference test was used to compare the two groups. A $\chi^{2}$ test was used for comparing qualitative data. A significant difference was set at a $\mathrm{P}<0.05$.

\section{Results}

\section{P. aeruginosa infected the wounds of diabetic rats}

\section{Wound healing}

Among the three diabetic groups, the $\mathrm{DM}+P$. aeruginosa group had more severe black callus, necrosis and erythematous on day 3 , with the most purulent secretion observed on day 7 . The diameter of the wound remained the greatest on day 21 compared to the $\mathrm{DM}+P$. aeruginosa $\triangle \mathrm{pcrV}$ and $\mathrm{DM}+$ uninfected groups (Figure 1). Among the three normal groups, there were more purulent secretions in the $\mathrm{N}+P$. aeruginosa group on the day 3 , with no obvious decrease in the wound diameter on day 7 . The wound diameter was clearly reduced on day 14 and the wound was nearly healed on day 21 . Compared with normal groups, the healing time of the diabetic groups was significantly delayed. On day 21, there was a tendency toward healing among the normal groups, while the wounds of the diabetic groups remained large. Compared with the $\mathrm{N}+P$. aeruginosa group, in the $\mathrm{DM}+P$. aeruginosa group there was black callus on day 3 , increased purulent secretions on day 7 , with no obvious reduction in the wound surface and continued purulent secretion with the wounds unhealed on day 21 .

\section{Colony growth in P. aeruginosa infected wounds}

Compared with the $\mathrm{N}+P$. aeruginosa group, the colony growth rate in the $\mathrm{DM}+P$. aeruginosa group was rapider $(\mathrm{P}<0.05)$, and the number of colonies in the $\mathrm{DM}+P$. aeruginosa group continued to increase until day 7 ; there was an inapparent decrease after day 7 . The colony number of the $\mathrm{DM}+P$. aeruginosa $\triangle \mathrm{pcrV}$ group began to decrease on day 3 . The colony number of both the $\mathrm{N}+P$. aeruginosa and $\mathrm{N}+$ P. aeruginosa $\triangle \mathrm{pcrV}$ groups continued to increase until day 3 then began to decrease. The rate of decrease in the $\mathrm{N}+$ P. aeruginosa $\triangle \mathrm{pcrV}$ group was more apparent and the number of colonies decreased significantly by day 21 . These results suggested that there was a persistent infection in the diabetic infection groups and the infection in the DM + $P$. aeruginosa group was the severest (Figure 2).

\section{Histopathology of wounds}

Infiltration of inflammatory cells was greater in $\mathrm{DM}+P$. aeruginosa group on days 7,14 than $\mathrm{DM}+P$. aeruginosa $\triangle$ pcrV group and DM + uninfected group. Infiltration of inflammatory cells was markedly reduced in normal groups, however, there were also many inflammatory cells in DM groups (Figure 3).

On day 0 , the skin tissue was intact and a large number of collagen fibers could be seen under the skin. On day 7 , there was a certain degree of collagen formation, but there was less collagen formation in diabetic wound infected by $P$. aeruginosa. However, the collagen in the wound without $P$. aeruginosa infection was more and denser. On day 14 , although there were abundant collagen fibers and fibroblasts 


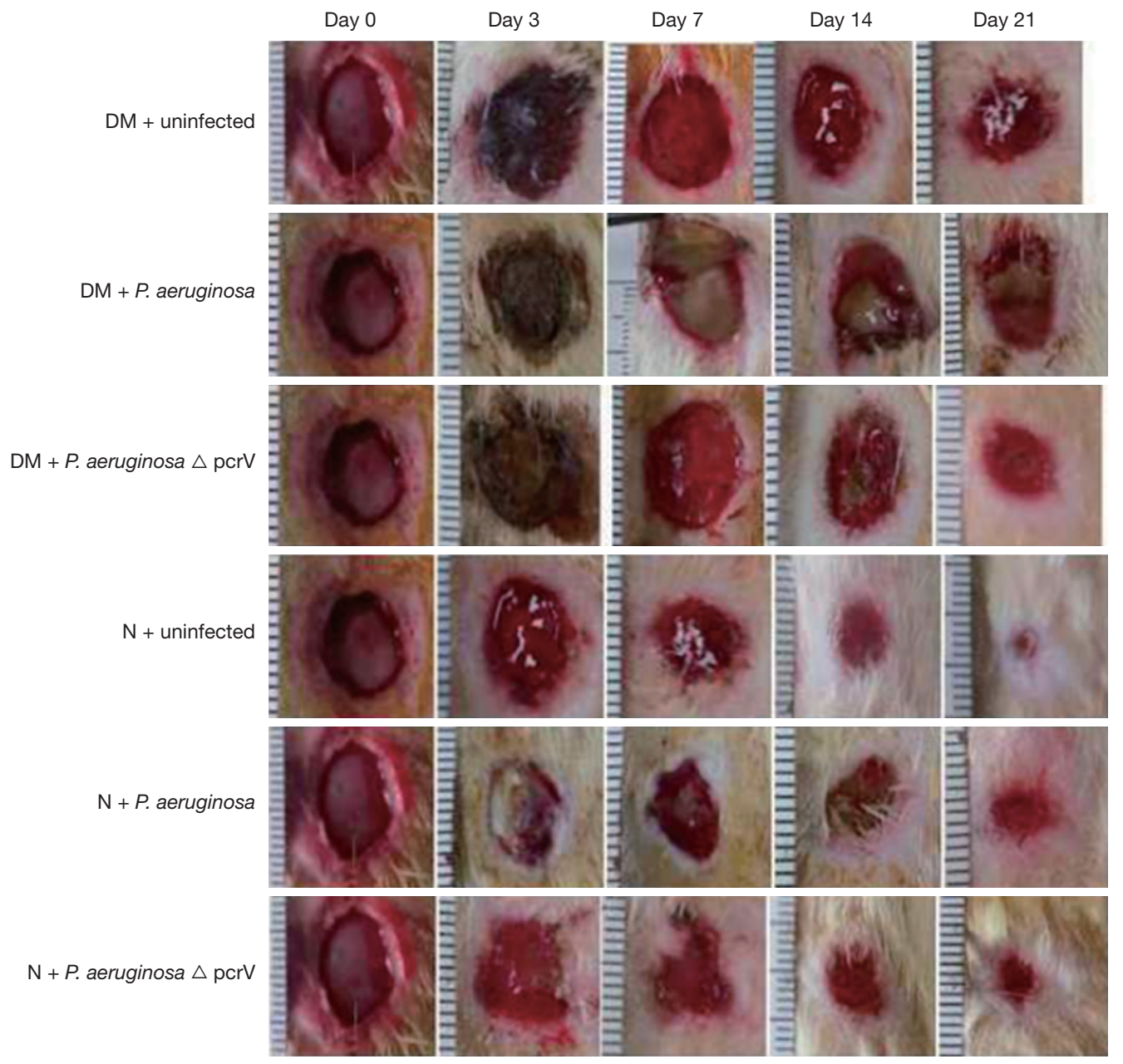

Figure 1 P. aeruginosa or P. aeruginosa $\triangle$ pcrV infected diabetic or normal rats back wounds. And there were two control groups (only wound or diabetic wound). Observing the progress of wound healing. In diabetic groups, P. aeruginosa infected group showed more serious black callus formation, necrosis and redness on day 3 , with the most purulent secretion observed on day 7 , the diameter of the wound was still the largest on day 21 compared with another two groups. In normal wounds, there were more purulent secretions in P. aeruginosa infected wound on the day 3 , with no obvious decrease in the wound on the day 7 ; the wound was obviously reduced on the day 14 , and the wound nearly healed on day 21 . On day 21 , there was a tendency toward healing among the normal wounds, while the diabetic wounds remained large. The condition of the wounds infected by $P$. aeruginosa $\triangle$ pcrV was better than that of the wounds infected by $P$. aeruginosa, however, was worse than normal wounds. DM, diabetic mellites; N, normal.

in the wound infected with $P$. aeruginosa, there were a few fibroblasts, mainly collagen fibers, arranged in order in the wound without $P$. aeruginosa, suggesting that the wound was mature (Figure 4).

\section{Expression of LC3-II/LC3-I, beclin-1 and p62}

Immunohistochemistry (Figures S1-S3, Table 1) and Western blot (Figure 5) analysis showed that the expression of LC3-II/LC3-I and beclin-1 increased gradually on days 0,7 and 14 in all groups, while the expression of p62 decreased gradually on days 7 and 14 . The expression of LC3-II/LC3-I and beclin-1 in the $\mathrm{N}+$ uninfected group was significantly higher than the uninfected diabetic group on days 7 and 14 (all $\mathrm{P}<0.05$ ), while the expression of p62 on days 7 and 14 was lower than the uninfected diabetic group (all $\mathrm{P}<0.05$ ). Among the diabetic infected rats, the expression of LC3-II/LC3-I and beclin-1 was higher in the $P$. aeruginosa $\triangle$ pcrV group than the $P$. aeruginosa group 


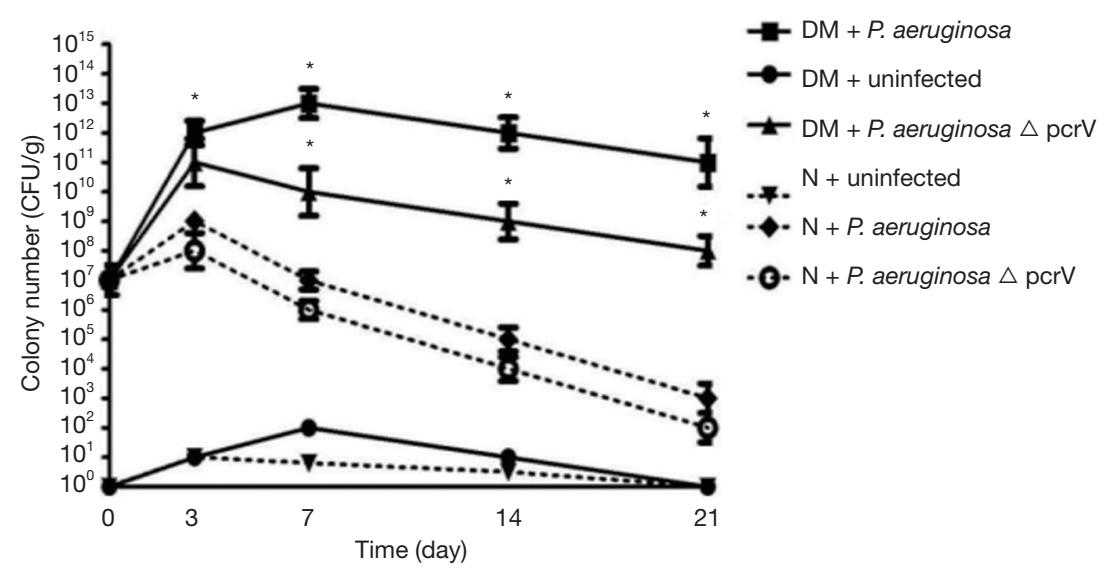

Figure 2 The colony growth rate in the diabetic wound infected with $P$. aeruginosa was faster than that in normal wound infected $P$. aeruginosa in the whole healing progress $\left({ }^{*} \mathrm{P}<0.05\right)$. The number of colonies in the diabetic wound infected with $P$. aeruginosa continued increasing until day 7. The colony number in the diabetic wound infected with $P$. aeruginosa $\triangle$ pcrV began decreasing on day 3 . In the normal infection wounds, the number of colonies was obvious less than that in diabetic infection wounds. DM, diabetic mellites; N, normal.

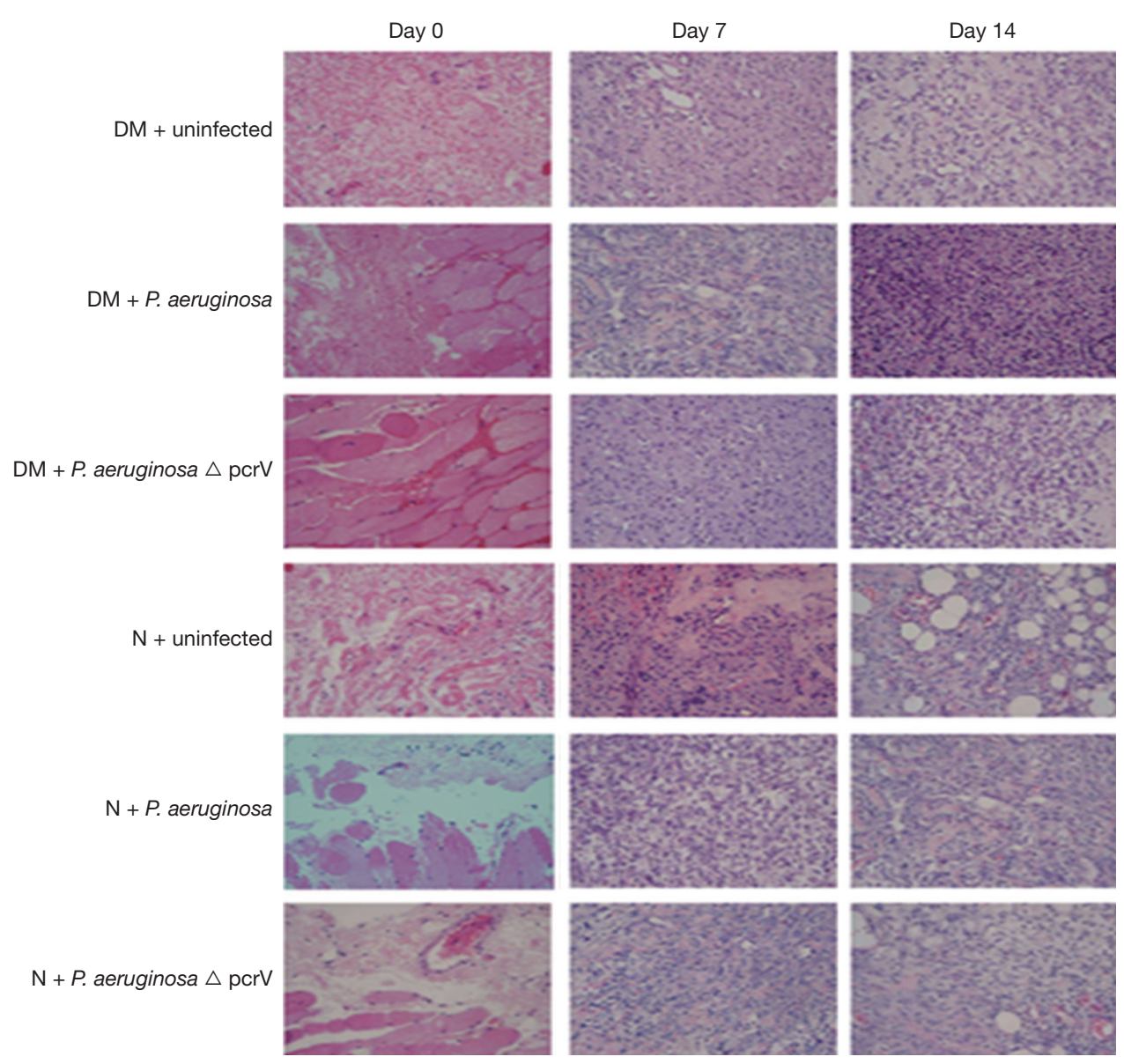

Figure 3 This was the histopathology of wounds. Infiltration of inflammatory cells was greater in DM $+P$. aeruginosa group on days 7,14 than other two groups. Infiltration of inflammatory cells was markedly reduced in normal groups, however, there were also many inflammatory cells in DM groups (400×, HE staining). 


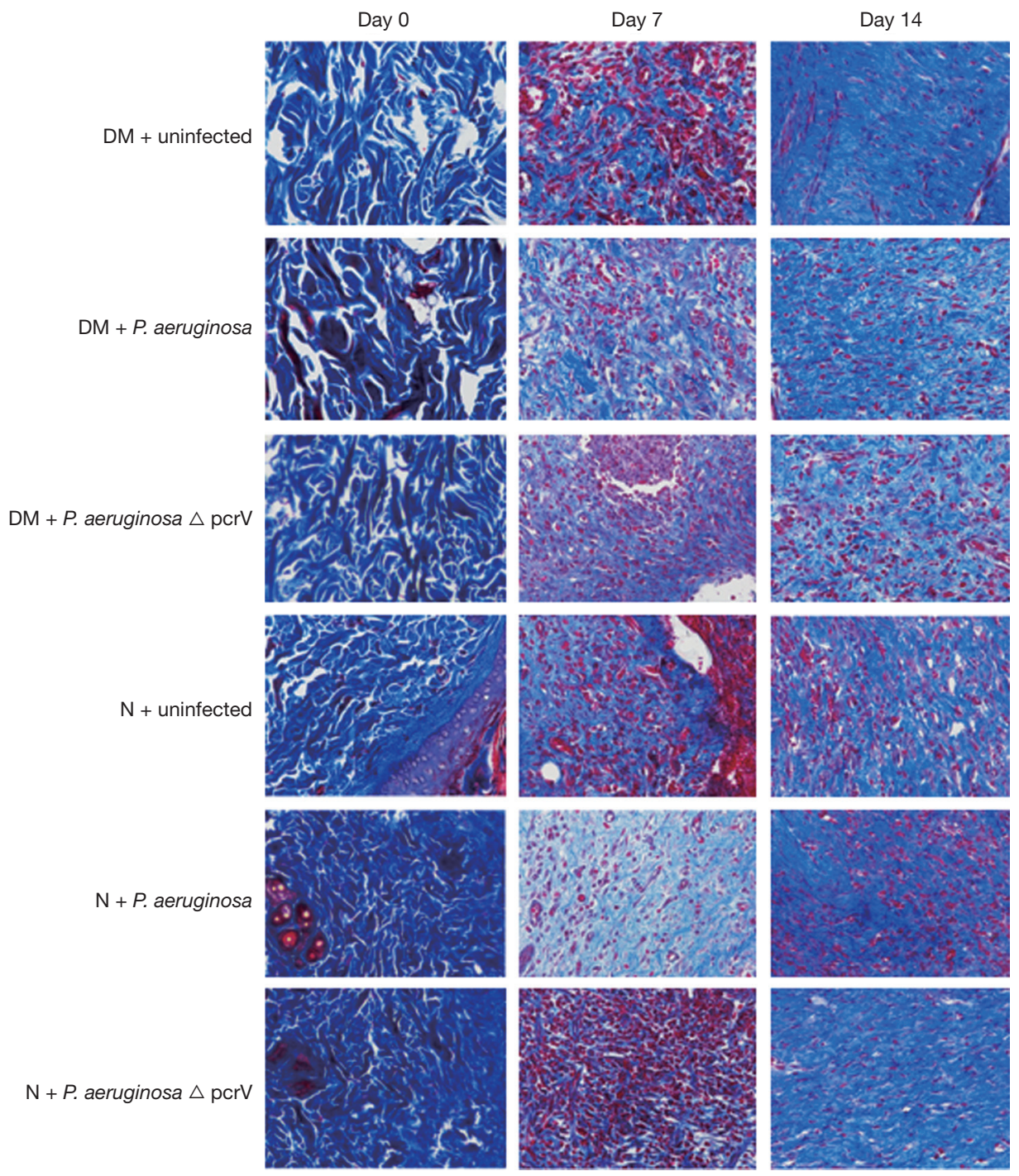

Figure 4 These showed the collagen changed in all groups. On day 0, the skin tissue was intact and a large number of collagen fibers. On day 7 , there was a certain degree of collagen formation, but there was less collagen formation in diabetic wound infected by $P$. aeruginosa. However, the collagen in the wound without $P$. aeruginosa infection was more and denser. On day 14, although there were abundant collagen fibers and fibroblasts in the wound infected with $P$. aeruginosa, there were a few fibroblasts, mainly collagen fibers, arranged in order in the wound without $P$. aeruginosa, suggesting that the wound was more mature. (400×, Masson staining).

on day 14 (all $\mathrm{P}<0.05)$, while the expression of p62 was lower than that of $P$. aeruginosa infected group (all $\mathrm{P}<0.05$ ). Compared with the $\mathrm{N}+P$. aeruginosa group the expression of LC3-II/LC3-I and beclin-1 in the DM $+P$. aeruginosa group was lower on day 14, while p62 expression was higher on day 14 (all $\mathrm{P}<0.05)$.

\section{Immunofluorescence results}

In the diabetic groups, the co-expression of macrophage specific markers (CD14 and LC3) in the DM + P. aeruginosa $\triangle$ pcrV group was greater than the $\mathrm{DM}+P$. aeruginosa group on day 14 (yellow fluorescence), suggesting that LC3 expression in macrophages in the $\mathrm{DM}+P$. aeruginosa $\triangle$ pcrV group was greater (Figure 6). In the normal groups, co-expression of CD14 and LC3 in $\mathrm{N}+P$. aeruginosa $\triangle$ pcrV group was greater than the $\mathrm{N}+P$. aeruginosa group on days 7 and 14 . 
Table 1 Mean optical density of Beclin-2, LC3, p62 at different time points in each group $(\bar{x} \pm \mathrm{s})$

\begin{tabular}{|c|c|c|c|c|c|c|c|c|c|}
\hline Groups & \multicolumn{3}{|c|}{ Beclin-1 } & \multicolumn{3}{|c|}{ LC3 } & \multicolumn{3}{|c|}{ p62 } \\
\hline DM + uninfected & $0.79 \pm 0.07$ & $1.51 \pm 0.06^{a}$ & $2.51 \pm 0.15^{\mathrm{a}}$ & $1.2 \pm 0.09$ & $2.35 \pm 0.13^{a}$ & $3.72 \pm 0.15^{\mathrm{a}}$ & $0.88 \pm 0.07$ & $2.72 \pm 0.19^{a}$ & $2.27 \pm 0.16^{\mathrm{a}}$ \\
\hline $\mathrm{N}+$ uninfected & $0.83 \pm 0.13$ & $2.32 \pm 0.14$ & $3.14 \pm 0.08$ & $1.25 \pm 0.14$ & $2.75 \pm 0.15$ & $4.26 \pm 0.19$ & $0.75 \pm 0.10$ & $1.80 \pm 0.14$ & $1.43 \pm 0.10$ \\
\hline $\mathrm{DM}+P$. aeruginosa & $0.87 \pm 0.14$ & $2.16 \pm 0.10$ & $2.80 \pm 0.07^{b}$ & $1.25 \pm 0.15$ & $2.96 \pm 0.08$ & $4.18 \pm 0.08^{b}$ & $1.18 \pm 0.15^{\mathrm{b}}$ & $2.7 \pm 0.18^{b}$ & $1.75 \pm 0.19^{b}$ \\
\hline $\begin{array}{l}\mathrm{DM}+P . \text { aeruginosa } \\
\triangle \mathrm{pcrV}\end{array}$ & $0.92 \pm 0.21$ & $2.2 \pm 0.09^{c}$ & $3.51 \pm 0.08^{\mathrm{c}, \mathrm{d}}$ & $1.23 \pm 0.10$ & $2.98 \pm 0.29^{c}$ & $4.71 \pm 0.15^{\mathrm{c}, \mathrm{d}}$ & $1.12 \pm 0.04^{c}$ & $2.68 \pm 0.15^{c}$ & $1.25 \pm 0.19^{\mathrm{c}, \mathrm{d}}$ \\
\hline $\begin{array}{l}\mathrm{N}+P . \text { aeruginosa } \triangle \\
\text { pcrV }\end{array}$ & $0.86 \pm 0.05$ & $2.75 \pm 0.19$ & $4.23 \pm 0.15$ & $1.31 \pm 0.15$ & $4.28 \pm 0.21$ & $5.36 \pm 0.23$ & $0.74 \pm 0.12$ & $1.20 \pm 0.17$ & $0.93 \pm 0.14$ \\
\hline
\end{tabular}

${ }^{a}$, vs. $\mathrm{N}+$ uninfected; ${ }^{\mathrm{b}}$, vs. $\mathrm{N}+P$. aeruginosa; ${ }^{\mathrm{c}}$, vs. $\mathrm{N}+P$. aeruginosa $\triangle \mathrm{pcrV} ;{ }^{\mathrm{d}}$, vs. $\mathrm{DM}+P$. aeruginosa. $\mathrm{N}$, normal; DM, diabetic mellitus; $P$. aeruginosa, Pseudomonas aeruginosa.
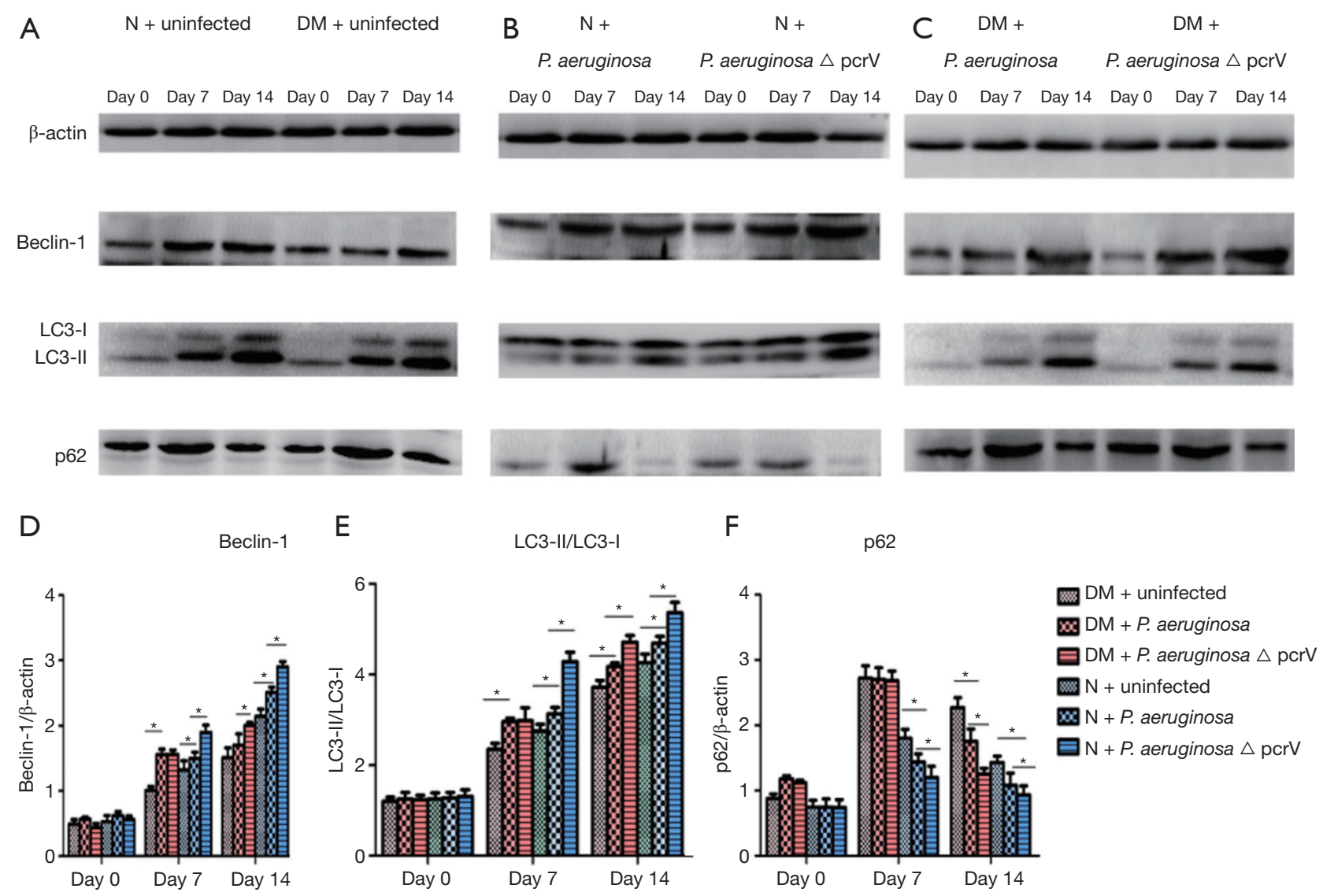

Figure 5 The expression of LC3-II/LC3-I and beclin-1 increased gradually on day 0, 7 and 14 in all groups, while the expression of p62 decreased gradually on days 7 and 14. Compared with normal wound infected with $P$. aeruginosa group, the expression of LC3 and beclin-1 in the diabetic wound infected with $P$. aeruginosa group was lower on day 14 , while p62 expression was higher on day $14\left({ }^{*} \mathrm{P}<0.05\right)$. In the diabetic infected wounds, the expression of LC3-II/LC3-I and beclin-1 was higher in the P. aeruginosa $\triangle$ pcrV group than that in $P$. aeruginosa group on day $14\left({ }^{*} \mathrm{P}<0.05\right)$, while the expression of $\mathrm{p} 62$ was lower than that in $P$. aeruginosa group $\left({ }^{*} \mathrm{P}<0.05\right)$. 


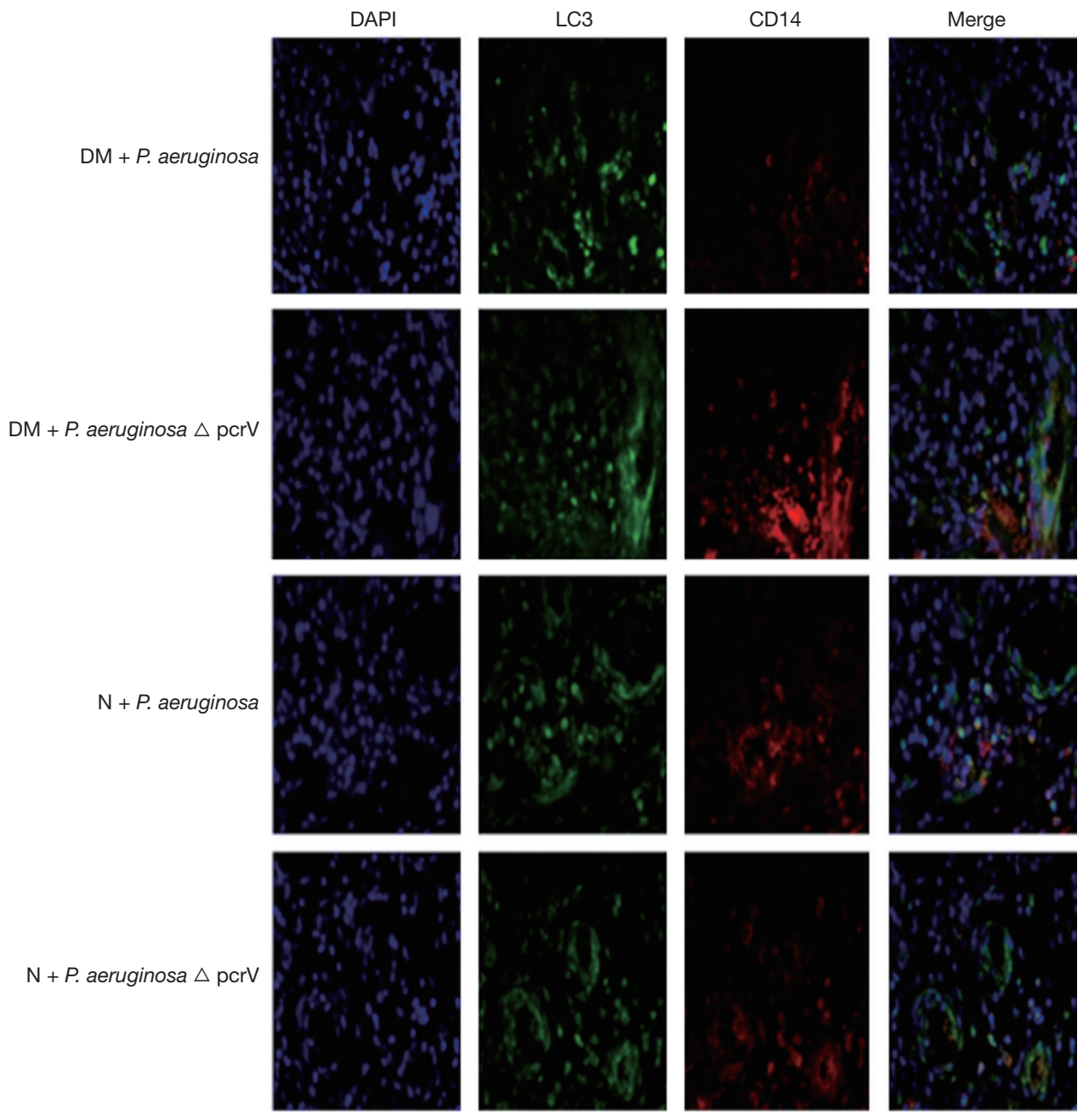

Figure 6 In diabetic wounds, the co-expression of macrophage-specific markers CD14 (red)and LC3(green) in P. aeruginosa $\triangle$ pcrV infecting group was higher than that in P. aeruginosa infecting group on day 14 (yellow). DAPI, blue stand for nucleus; LC3, red stand for LC3; CD14, green stand for macrophage; DM, diabetic mellitus; N, normal.

\section{Effects of rapamycin-induced autophagy on the wounds of diabetic rats infected with $P$. aeruginosa}

\section{Wound healing}

Purulent secretion in the $\mathrm{DM}+P$. aeruginosa + rapamycin group decreased on day 7 compared with the $\mathrm{DM}+P$. aeruginosa group, and the diameter of the wound decreased significantly by day 14 (Figure 7). Compared with the $\mathrm{N}+$ $P$. aeruginosa group, tissue rupture was milder and the purulent secretion was less in the $\mathrm{N}+P$. aeruginosa + rapamycin group and the diameter of the wound was not enlarged on day 3. The diameter of the wound was clearly reduced on day 7 and the wound was nearly healing by day 21. Compared with the $\mathrm{DM}+P$. aeruginosa group, the diameter of the wound was distinctly smaller on day 7 and the purulent secretion was significantly decreased in the $\mathrm{DM}+P$. aeruginosa + gentamicin group. Compared with the $\mathrm{N}+P$. aeruginosa group-the necrosis was mild on day 3 and the wound was basically healing by day 21 in the $\mathrm{N}+P$. aeruginosa + gentamicin group.

\section{$P$. aeruginosa colony growth by inducing autophagy}

The number of colonies in the $\mathrm{DM}+P$. aeruginosa and the $\mathrm{DM}+P$. aeruginosa + rapamycin groups raising up to day 7 ; however, the rate of increase in the $\mathrm{DM}+P$. aeruginosa + rapamycin group was slower and the number of colonies began to decrease after day $7(\mathrm{P}<0.05)$. Among the normal 


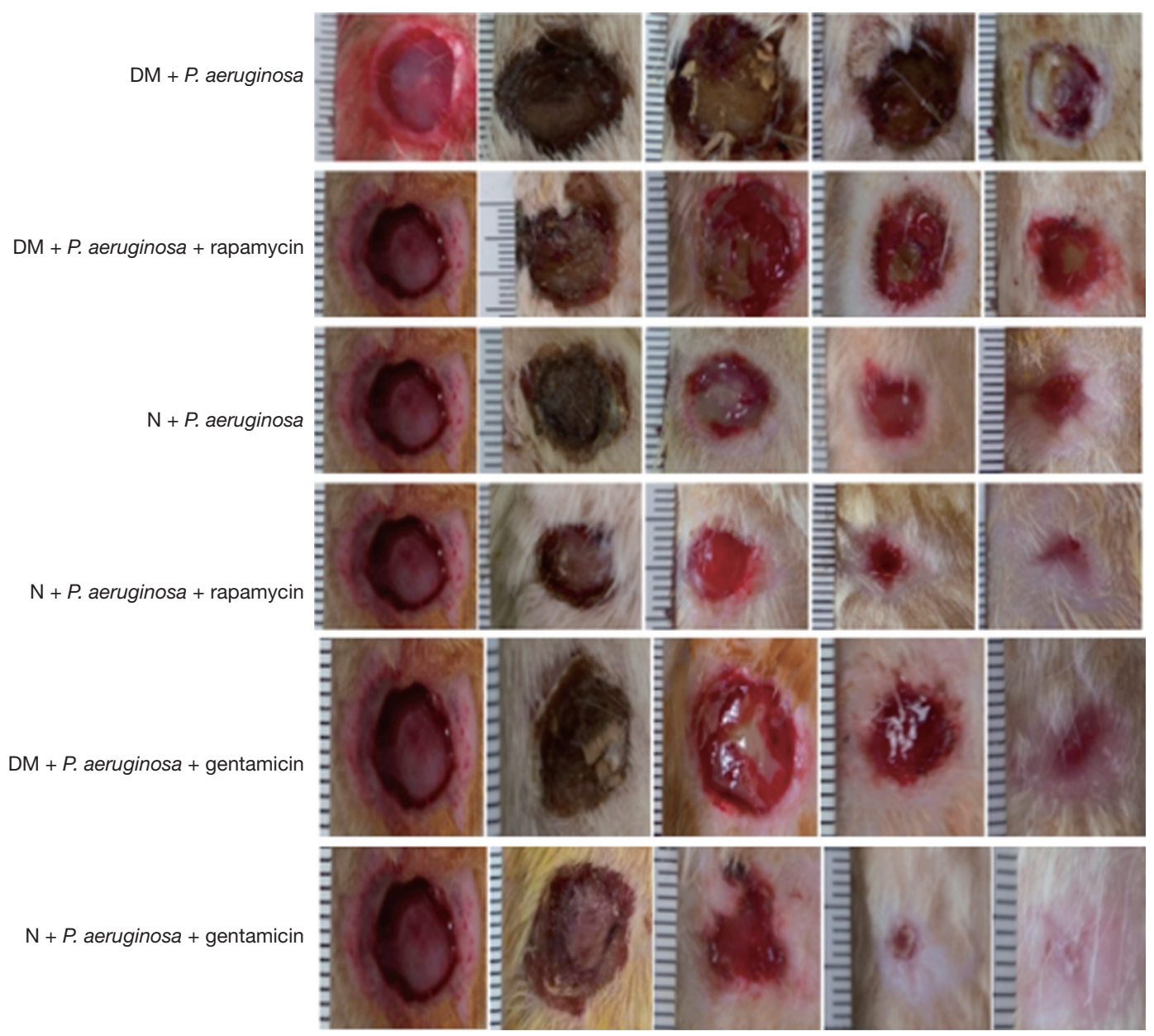

Figure 7 Purulent secretion in diabetic wound infected with $P$. aeruginosa and interfered with rapamycin was decreased on day 7 compared with diabetic wound infected with $P$. aeruginosa without rapamycin, and the area of the wound had decreased significantly by day 14 . Compared with the diabetic wound infected with $P$. aeruginosa. The area of the wound was significantly smaller on day 7 , and the purulent secretion was significantly decreased in the diabetic wound infected with P. aeruginosa and interfered with Gentamicin, compared with the diabetic wound infected with P. aeruginosa.

infection groups, the number of colonies in the $\mathrm{N}+P$. aeruginosa + rapamycin group was evidently decreased and the clearance of PA was increased on day 3 compared with the $\mathrm{N}+P$. aeruginosa group $(\mathrm{P}<0.05)$. In the $\mathrm{DM}+P$. aeruginosa + gentamicin group, the rate of increase in the number of colonies was slower, and the number of colonies began to decrease after 7 days compared with the $\mathrm{DM}+P$. aeruginosa group. Among the normal infection groups, the number of colonies in the $\mathrm{N}+P$. aeruginosa + gentamicin group started to increase slowly until day 3 and decreased distinctly thereafter (Figure 8).

\section{Histopathology of wounds}

Compared with the $\mathrm{DM}+P$. aeruginosa and $\mathrm{DM}+$
P. aeruginosa + rapamycin groups, the infiltration of inflammatory cells decreased on days 7 and 14 in DM+ $P$. aeruginosa +gentamicin group. There was similar characteristics in normal groups (Figure 9).

\section{Expression of LC3-II/LC3-I, beclin-1 and p62 by inducing autophagy}

Immunohistochemistry (Figures S4-S6, Table 2) and Western blot (Figure 10) analysis showed that among the diabetic groups, the expression of LC3-II/LC3-I was higher on days 7 and 14 the expression of beclin-1 was higher on day 14 (all $\mathrm{P}<0.05)$, and the expression of p62 was lower on days 7 and 14 in the $\mathrm{DM}+P$. aeruginosa + rapamycin group compared with the $\mathrm{DM}+P$. aeruginosa group (all 
A

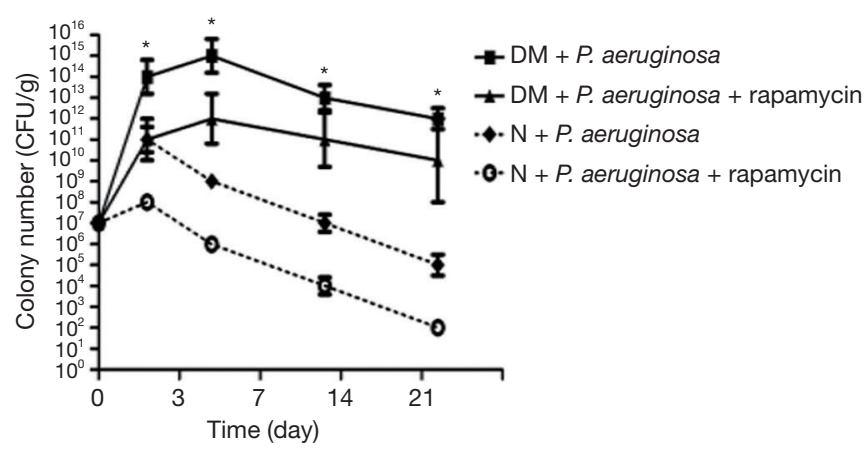

B

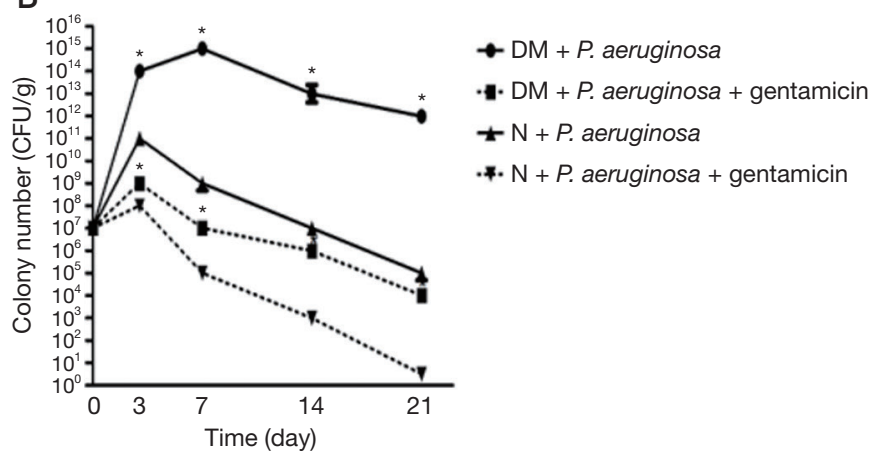

Figure 8 The colony growth rate was faster in the diabetic wounds infected $P$. aeruginos $a$ than that in the normal wound infected with $P$. aeruginosa $\left({ }^{*} \mathrm{P}<0.05\right)$. The colony numbers in the diabetic wounds increased up to day 7 ; however, the growth rate in the diabetic wound infected with $P$. aeruginosa and interfered with rapamycin was slower than that without rapamycin, and the number of colonies began to decrease after day $7\left({ }^{*} \mathrm{P}<0.05\right)$. Among the normal wounds infected with $P$. aeruginosa, the colony number in the normal wounds with $P$. aeruginosa and interfered with rapamycin was significantly decreased and the clearance of $P$. aeruginosa was increased on day 3, compared with that in the normal wounds infected with P. aeruginosa $\left({ }^{*} \mathrm{P}<0.05\right)$. In the gentamicin interfered wounds, the rate of increase in colony number was slower, and the number of colonies began to decrease after 7 days in diabetic wound, compared with the wound without gentamicin. Among the normal wounds infected with P. aeruginosa and interfered with gentamicin group began to increase slowly until day 3 , and decreased significantly thereafter.

$\mathrm{P}<0.05)$. There was no obvious difference in the expression of beclin-1, LC3-II/LC3-I and p62 between the DM $+P$. aeruginosa + gentamicin and $\mathrm{DM}+P$. aeruginosa groups at any time point (all $\mathrm{P}>0.05$ ). The expression of LC3-II/ LC3-I and beclin-1 was greater and the expression of p62 was less on days 7 and day 14 in the $\mathrm{N}+P$. aeruginosa + rapamycin group compared with the $\mathrm{DM}+P$. aeruginosa + rapamycin group.

\section{Discussion}

DFI can lead to the deterioration of diabetic foot, an increased amputation rate and even death (11-14). The principle of antimicrobial stewardship is to control infection effectively in minimum days $(15,16)$. More prolonged antibiotic therapy is associated with increased risks of adverse events, greater disruption of host microbiomes, higher costs and more patient inconvenience. More importantly, there will be a large number of resistant strains, such as MRSA and MDRPA. The proportion of MDRPA infection has been increasing, however, the research and development of new antibiotics is slow, so we should find new treatment approaches.

Autophagy can clear microbes and moderate host innate immune responses to microbial products. The direct elimination of microbes by autophagy (a process termed xenophagy) has received the most attention. Indeed, xenophagy plays an outstanding host protective role in infection $(17,18)$.

Beclin-1 is an upstream signal that acts at the initiation stage of autophagy, first binding to the membrane of the phagocytic vesicle, which is formed by enveloping the material targeted for degradation in a bilayer membrane, and then recruiting other autophagy-associated proteins to initiate downstream signals (19). In our study, autophagy occurred in the cells of the wound, but the level of belin-1 in rats with diabetes was lower than normal rats, suggesting that hyperglycemia could inhibit the initiation of autophagy. After $P$. aeruginosa infection, in order to clear P. aeruginosa, autophagy in wound should increase, which is also a significant way to regulate homeostasis. However, we found that the initiation of autophagy in $P$. aeruginosa with T3SS was weaker than $P$. aeruginosa without T3SS, suggesting that T3SS of $P$. aeruginosa had an inhibitory effect on the initiation of autophagy.

The content of LC3-II in a tissue sample is proportional to the number of autophagosomes, and thus reflects autophagic flux (20,21). As autophagy progress, LC3-I transforms into LC3-II and the ratio of LC3-II/LC3-I increases, suggesting the formation of autophagosome. We found that the LC3-II/LC3-I was less in DM $+P$. aeruginosa group, suggesting that $P$. aeruginosa also inhibited 


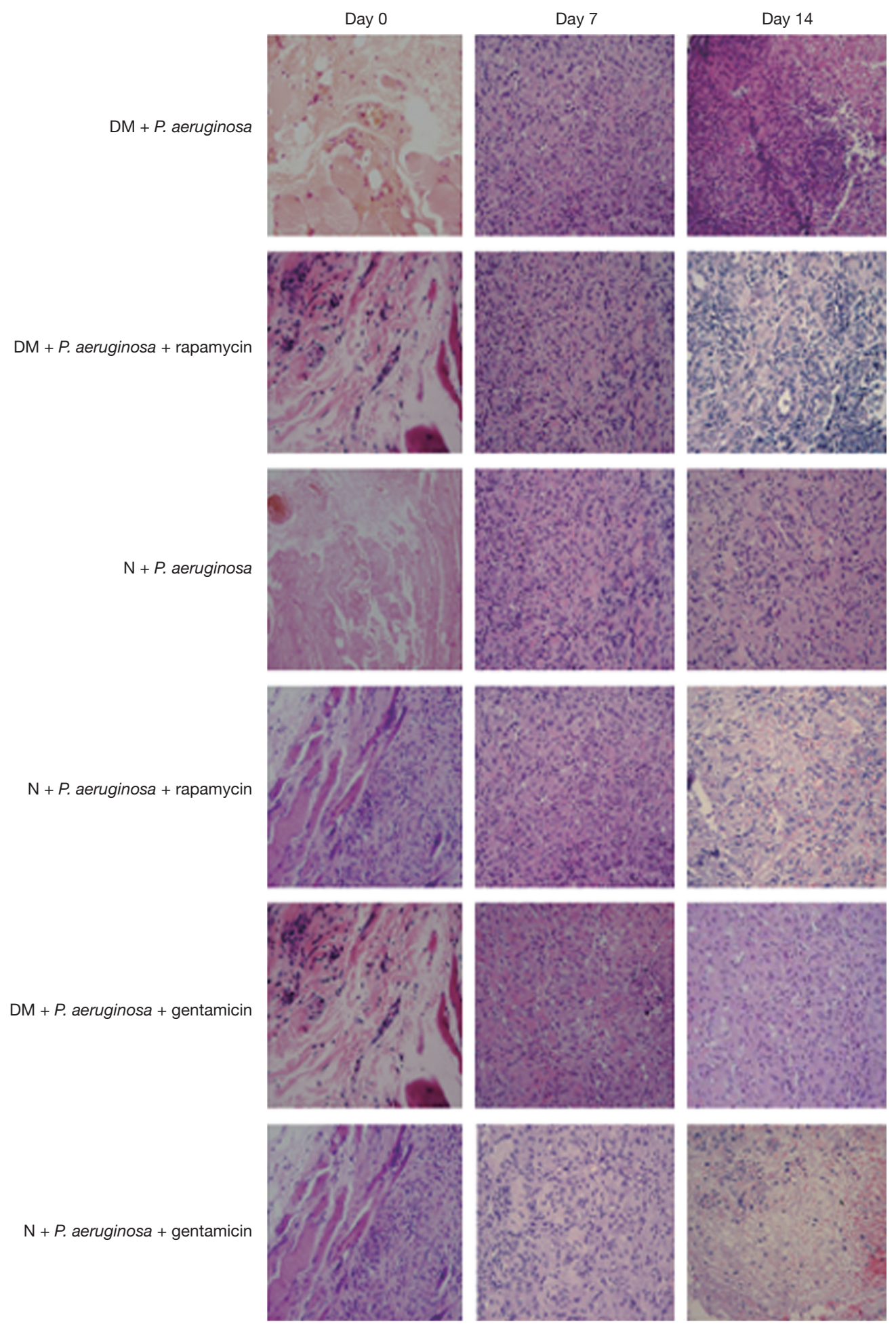

Figure 9 Compared with the DM + P. aeruginosa and DM + P. aeruginosa + rapamycin groups, the infiltration of inflammatory cells had decreased on days 7 and 14 in DM+ P. aeruginosa + Gentamicin group. There was similar characteristics in normal groups (400× HE staining). 
Table 2 Mean optical density of Beclin-2, LC3, p62 at different time points in each group $(\bar{x} \pm \mathrm{s})$

\begin{tabular}{|c|c|c|c|c|c|c|c|c|c|}
\hline Groups & \multicolumn{3}{|c|}{ Beclin-1 } & \multicolumn{3}{|c|}{ LC 3} & \multicolumn{3}{|c|}{ p62 } \\
\hline $\mathrm{DM}+P$. aeruginosa & $0.86 \pm 0.14$ & $2.18 \pm 0.10$ & $3.20 \pm 0.12$ & $1.28 \pm 0.10$ & $2.78 \pm 0.20$ & $3.72 \pm 0.15$ & $0.95 \pm 0.18$ & $2.72 \pm 0.15$ & $1.75 \pm 0.10$ \\
\hline $\begin{array}{l}\mathrm{DM}+P . \text { aeruginosa } \\
+ \text { rapamycin }\end{array}$ & $0.92 \pm 0.21$ & $2.25 \pm 0.10$ & $3.80 \pm 0.14^{a}$ & $1.25 \pm 0.08$ & $3.53 \pm 0.08^{a}$ & $4.94 \pm 0.47^{\mathrm{a}}$ & $0.85 \pm 0.19$ & $1.95 \pm 0.15^{\mathrm{a}}$ & $1.33 \pm 0.16^{a}$ \\
\hline $\begin{array}{l}\mathrm{DM}+P . \text { aeruginosa } \\
+ \text { gentamicin }\end{array}$ & $0.90 \pm 0.22$ & $2.24 \pm 0.14$ & $3.25 \pm 0.17$ & $1.24 \pm 0.09$ & $3.04 \pm 0.12$ & $3.88 \pm 0.21$ & $0.89 \pm 0.19$ & $2.64 \pm 0.13$ & $1.82 \pm 0.16$ \\
\hline $\begin{array}{l}\mathrm{N}+P . \text { aeruginosa }+ \\
\text { gentamicin }\end{array}$ & $0.88 \pm 0.07$ & $2.62 \pm 0.09$ & $4.29 \pm 0.15$ & $1.95 \pm 0.19$ & $3.82 \pm 0.17$ & $4.75 \pm 0.14$ & $0.88 \pm 0.14$ & $1.69 \pm 0.16$ & $0.84 \pm 0.11$ \\
\hline
\end{tabular}

${ }^{a}$, vs. DM + P. aeruginosa; ${ }^{b}$, vs. $\mathrm{N}+P$. aeruginosa; ${ }^{\mathrm{c}}, \mathrm{vs} . \mathrm{DM}+P$. aeruginosa + rapamycin. $\mathrm{N}$, normal; DM, diabetic mellitus; $P$. aeruginosa, Pseudomonas aeruginosa.

translocation of LC3-I thereby reducing the formation of autophagosome or autophagic flux. It was also found that the formation of autophagosome in macrophages was reduced by fluorescent staining. As a result, the number of $P$. aeruginosa colonies in the wound increased, the symptoms were severe, and the healing was slow.

The ubiquitin-binding domain of p62 first binds to the substrate to be degraded and then interacts with the LC3 protein located on the pre-autophagy membrane. Finally, the ubiquitinated substrate bound to p62 enters the mature autophagosome and is degraded by the lysosome (22). The decline in p62 suggests formation of autolysosome (23). We showed that the level of $\mathrm{p} 62$ was more in the $\mathrm{DM}+P$. aeruginosa group, suggesting that $P$. aeruginosa also inhibited the formation of autolysosomes.

In hyperglycemia and the normal environment, when the cutaneous wound occurred in rats, autophagy will occur in the body to promote wound healing; however, a high glucose environment could inhibit the entire process, (including initiation, formation of autophagosome and autolysosome) then healing slowed (including the speed and quality of wound healing). These findings were consistent with the results of Chen et al. (24), who found that a decreased level of autophagy in endothelial progenitor cells in the peripheral blood of patients with diabetic foot ulcers associated with hyperglycemia.

Yuan et al. (25) reported that infection of alveolar macrophages with the $P$. aeruginosa, which had an intact T3SS, induced autophagy facilitated the clearance of $P$. aeruginosa. Our results shown that the inhibition of the whole process of autophagy in $P$. aeruginosa with T3SS was stronger than without T3SS. P. aeruginosa is an extracellular bacterium, the "needle-like" structure of T3SS is inserted into the cell membrane of the host and the virulence factor is injected into the cell to destroy the host cell (25-27). We inferred that the virulence factors of T3SS must interact with the key molecules in the autophagy pathway in the cell, leading to slowing of autophagy initiation and a reduction in autophagosome and autolysosome thus inhibiting the entire process of autophagy. When the T3SS of $P$. aeruginosa was knocked out and the full process of autophagy was restored partly.

$P$. aeruginosa weakened the whole process of autophagy, we tried to eliminate $P$. aeruginosa by increasing autophagy level of the host. Rapamycin is a classic autophagy inducer that specifically inhibits the activity of mammalian target of rapamycin (mTOR) kinases, preventing the phosphorylation of downstream target proteins thereby enhancing autophagy (28). We found that rapamycin strengthened $P$. aeruginosa clearance by increasing autophagy in both normal and diabetic wounds in rats. Thus, autophagy induced by rapamycin achieved the goal of effective sterilization and its bactericidal effect was equivalent to gentamicin.

In our research, it was clear that T3SS of $P$. aeruginosa inhibited the entire process of autophagy of wound cells so that the infectious process continued and wound healing was delayed. The specific sites and mechanisms of effective proteins of T3SS acting with beclin-1, LC3 and p62 have not been elucidated and may also interact with other 


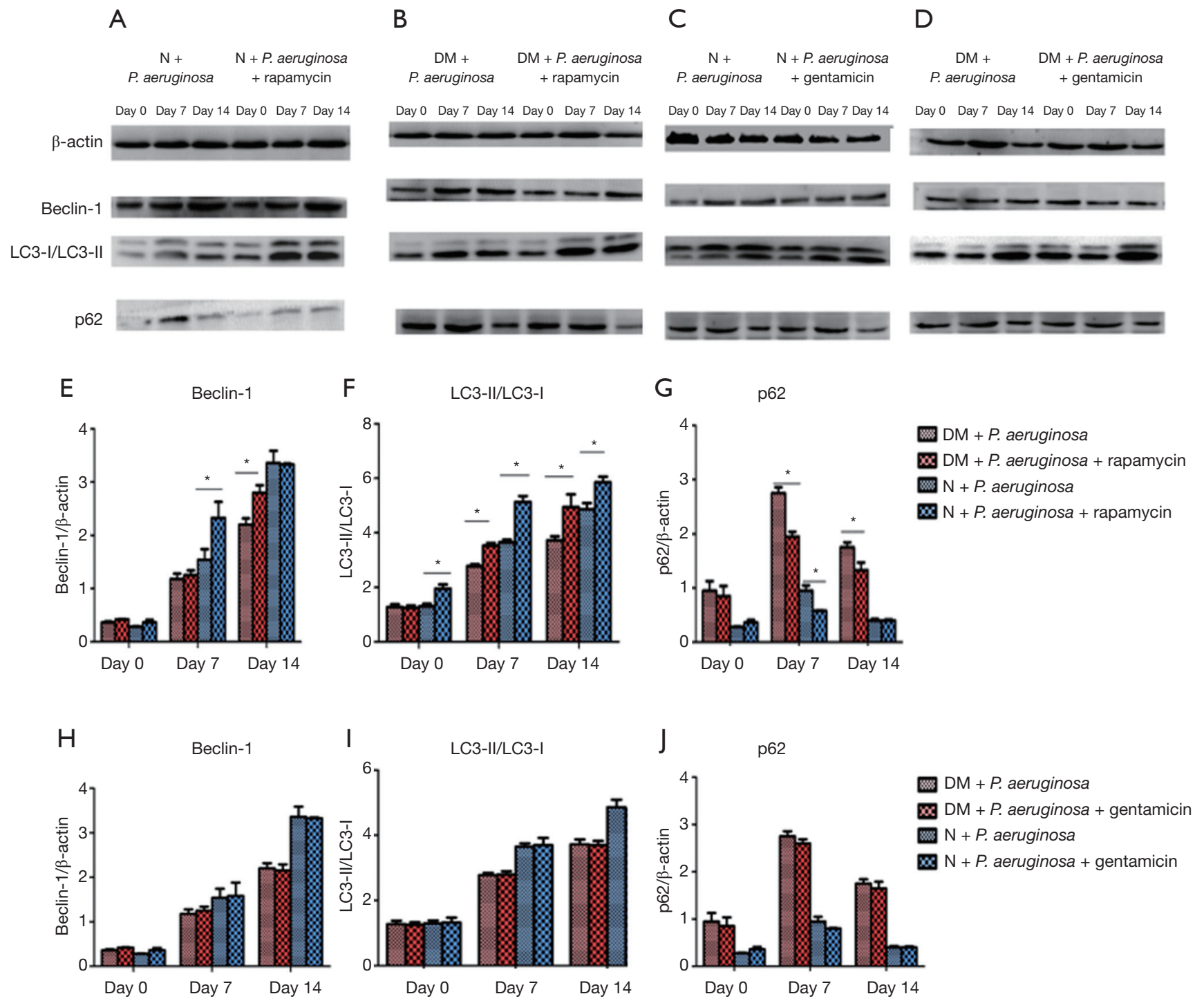

Figure 10 Among the diabetic wounds, after rapamycin inducing, the expression of LC3-II/LC3-I was higher on day 7 and 14, the expression of beclin-1 was higher on day 14 (all ${ }^{*} \mathrm{P}<0.05$ ), and the expression of $\mathrm{p} 62$ was lower on day 7 and 14 , compared without rapamycin $\left({ }^{*} \mathrm{P}<0.05\right)$. There was no significant expression difference in the wound interfered with gentamicin and without gentamicin $(\mathrm{P}>0.05)$. Among the normal wounds, there were similar expression. However, the expression of LC3-II/LC3-I, beclin-1 were lower and the expression of p62 were higher on day 7 and day 14 in diabetic wounds infected with P. aeruginosa and induced with rapamycin than that in corresponding normal wounds.

molecules in the autophagy signaling pathway. All of the above will be our further research objectives. The most outstanding findings for treatment was enhancing autophagy could effectively kill bacteria. The mechanism is completely different from of antibiotics. When there are antibioticresistant bacteria, especially multiple antibiotic-resistant bacteria, we can use the new strategy to eliminate bacteria.
Autophagy cannot only remove $P$. aeruginosa, but also other bacteria, such as Staphylococcus aureus (29). Autophagy has a great prospect in the clinical research of DFI.

\section{Acknowledgments}

Funding: This work was supported by Natural Science 
Foundation of China (81670766).

\section{Footnote}

Reporting Checklist: The authors have completed the ARRIVE reporting checklist. Available at http://dx.doi. org/10.21037/atm-20-1019

Conflicts of Interest: All authors have completed the ICMJE uniform disclosure form (available at http://dx.doi. org/10.21037/atm-20-1019). The authors have no conflicts of interest to declare.

Ethical Statement: The authors are accountable for all aspects of the work in ensuring that questions related to the accuracy or integrity of any part of the work are appropriately investigated and resolved. Experiments were performed under a project license (No.: SCXK20190008) granted by the Ethics Committee of Tianjin Medical University, in compliance with the guidelines of Ethics Committee of Tianjin Medical University for the care and use of animals.

Open Access Statement: This is an Open Access article distributed in accordance with the Creative Commons Attribution-NonCommercial-NoDerivs 4.0 International License (CC BY-NC-ND 4.0), which permits the noncommercial replication and distribution of the article with the strict proviso that no changes or edits are made and the original work is properly cited (including links to both the formal publication through the relevant DOI and the license). See: https://creativecommons.org/licenses/by-nc-nd/4.0/.

\section{References}

1. International Diabetes Federation. IDF Diabetes Atlas. Sixth edition, 2013.

2. Whiting DR, Guariguata L, Weil C, et al. IDF diabetes atlas: global estimates of the prevalence of diabetes for 2011 and 2030. Diabetes Res Clin Pract 2011;94:311-21.

3. Bansal E, Garg A, Bhatia S, et al. Spectrum of microbial flora in diabetic foot ulcers. Indian J Pathol Microbiol 2008;51:204-8.

4. Yoga R, Khairul A, Sunita K, et al. Bacteriology of diabetic foot lesions. Med J Malaysia 2006:61 Suppl A:14-6.

5. Shakil S, Khan AU. Infected foot ulcers in male and female diabetic patients: a clinico-bioinformative study. Ann Clin Microbiol Antimicrob 2010;9:2.
6. Li X, Qi X, Yuan G, et al. Microbiological profile and clinical characteristics of diabetic foot infection in northern China: a retrospective multicenter survey in the Beijing area. J Med Microbiol 2018;67:160-8.

7. Brutinel ED, Vakulskas CA, Yahr TL. ExsD inhibits expression of the Pseudomonas aeruginosa type III secretion system by disrupting ExsA self-association and DNA binding activity. J Bacteriol 2010;192:1479-86.

8. El Solh AA, Akinnusi ME, Wiener-Kronish JP, et al. Persistent infection with Pseudomonas aeruginosa in ventilator-associated pneumonia. Am J Respir Crit Care Med 2008;178:513-19.

9. Morris MC, Gilliam EA, Li L. Innate immune programing by endotoxin and its pathological consequences. Front Immunol 2015;5:680.

10. Ma Y, Hou X, Zhu X, et al. Autophagy-related protein expressions in wound granulation tissues from diabetic foot patients infected with Pseudomonas aeruginosa. Chinese Journal of Endocrinology and Metabolism 2017;33:23-8.

11. Peters EJ, Lipsky BA. Diagnosis and management of infection in the diabetic foot. Med Clin North Am 2013;97:911-46.

12. Lavery LA, Armstrong DG, Murdoch DP, et al. Validation of the Infectious Diseases Society of America's diabetic foot infection classification system. Clin Infect Dis 2007;44:562-5.

13. Ndosi M, Wright-Hughes A, Brown S, et al. Prognosis of the infected diabetic foot ulcer: a 12-month prospective observational study. Diabet Med 2018;35:78-88.

14. Tan TW, Shih CD, Concha-Moore KC, et al. Disparities in outcomes of patients admitted with diabetic foot infections. PLoS One 2019;14:e0211481.

15. Lipsky BA, Dryden M, Gottrup F, et al. Antimicrobial stewardship in wound care: a Position Paper from the British Society for Antimicrobial Chemotherapy and European Wound Management Association. J Antimicrob Chemother 2016;71:3026-35.

16. Uçkay I, Berli M, Sendi P, et al. Principles and practice of antibiotic stewardship in the management of diabetic foot infections. Curr Opin Infect Dis 2019;32:95-101.

17. Deretic V, Kimura G. Timmins, et al. Immunologic manifestations of autophagy. J Clin Invest 2015;125:75-84.

18. Rubinsztein DC, Bento CF, Deretic V. Therapeutic targeting of autophagy in neurodegenerative and infectious diseases. J Exp Med 2015;212:979-90.

19. Pyo JO, Nah J, Jung YK. Molecules and their functions in autophagy. Exp Mol Med 2012;44:73-80.

20. Kabeya Y, Mizushima N, Ueno T, et al. LC3, a mammalian 
homologue of yeast Apg8p, is localized in autophagosome membranes after processing. EMBO J 2000;19:5720-8.

21. Klionsky DJ, Abdelmohsen K, Abe A, et al. Guidelines for the use and interpretation of assays for monitoring autophagy (3rd edition). Autophagy 2016;12:1-222.

22. Mathew R, Karp CM, Beaudoin B, et al. Autophagy suppresses tumorigenesis through elimination of p62. Cell 2009; 137:1062-75.

23. Lin X, Li S, Zhao Y, et al. Interaction domains of p62: a bridge between p62 and selective autophagy. DNA Cell Biol 2013;32:220-7.

24. Chen W, Wu Y, Li L, et al. Adenosine accelerates the healing of diabetic ischemic ulcers by improving autophagy of endothelial progenitor cells grown on a biomaterial. Sci Rep 2015,5:11594.

25. Yuan K, Huang C, Fox J, et al. Autophagy plays an

Cite this article as: $\mathrm{Xu} \mathrm{J,} \mathrm{Ma} \mathrm{Y,} \mathrm{Zhu} \mathrm{X,} \mathrm{Zhang} \mathrm{J,} \mathrm{Cheng} \mathrm{Z,}$ Wu W, Wang P. Enhanced autophagy promotes the clearance of Pseudomonas aeruginosa in diabetic rats with wounds. Ann Transl Med 2020;8(21):1362. doi: 10.21037/atm-20-1019 essential role in the clearance of Pseudomonas aeruginosa by alveolar macrophages. J Cell Sci 2012;125:507-15.

26. Jia J, Alaoui-El-Azher M, Chow M, et al. c-Jun NH2terminal kinase-mediated signaling is essential for Pseudomonas aeruginosa ExoS-induced apoptosis. Infect Immun.2003,71:3361-70.

27. He SY, Nomura K, Whittam TS. Type III protein secretion mechanism in mammalian and plant pathogens. Biochim Biophys Acta 2004;1694:181-206.

28. Kamada Y, Sekito T, Ohsumi Y. Autophagy in yeast: a TOR-mediated response to nutrient starvation. Curr Top Microbiol Immunol 2004;279:73-84.

29. Maurer K, Reyes-Robles T, Alonzo F 3rd, et al. Autophagy mediates tolerance to Staphylococcusaureus alpha-toxin. Cell Host Microbe 2015;17:429-40. 